\title{
Precision measurements of cosmic rays up to the highest energies with a large radio array at the Pierre Auger Observatory
}

\author{
Jörg R. Hörandel ${ }^{1,2,3, *}$ for the Pierre Auger Collaboration ${ }^{4, * *}$ \\ ${ }^{1}$ Radboud Universiteit, Department of Astrophysics/IMAPP, P.O. Box 9010, 6500 GL Nijmegen, The Netherlands \\ ${ }^{2}$ Nikhef, Science Park 105, 1098 XG Amsterdam, The Netherlands \\ ${ }^{3}$ Vrije Universiteit Brussel, Department of Physics and Astronomy, B-1050 Brussels, Belgium \\ ${ }^{4}$ Observatorio Pierre Auger, Av. San Martin Norte 304, 5613 Malargüe, Argentina
}

\begin{abstract}
The Pierre Auger Observatory is presently being upgraded to enlarge its detection capabilities for ultra-high-energy cosmic rays. Part of this upgrade is a radio detector array, aimed to cover a surface area of $3000 \mathrm{~km}^{2}$ to measure the properties of the highest-energy cosmic rays. The plans for this radio upgrade are outlined.
\end{abstract}

\section{Introduction}

The Pierre Auger Observatory, located in western Argentina, is the world's largest cosmic-ray Observatory [1]. The objectives of the Observatory are to probe the origin and characteristics of cosmic rays above $10^{17} \mathrm{eV}$ and to study the interactions of these, the most energetic particles observed in Nature. The Auger design features an array of 1600 water-Cherenkov detector stations on a $1500 \mathrm{~m}$ grid, spread over $3000 \mathrm{~km}^{2}$, and overlooked by 24 air fluorescence telescopes. The layout is illustrated in Fig. 1. In addition, three high-elevation fluorescence telescopes overlook a $23.5 \mathrm{~km}^{2}, 61$-detector infilled array with $750 \mathrm{~m}$ spacing. Radio emission from extensive air showers is measured with the Auger Engineering Radio Array (AERA), comprising more than 150 radio detector stations, covering an area of about $17 \mathrm{~km}^{2}$, co-located with the infill array $[2,3]$.

At present, the Pierre Auger Collaboration is working on an upgrade of the Observatory, Auger Prime [4]. The physics case of the upgrade is outlined in [5]. The key science questions to be addressed are: What are the sources and acceleration mechanisms of ultra-high-energy cosmic rays (UHECRs)? Do we understand particle acceleration and physics at energies well beyond the LHC (Large Hadron Collider) scale? What is the fraction of protons, photons, and neutrinos in cosmic rays at the highest energies?

Objectives of the upgrade are to:

- Elucidate the mass composition and the origin of the flux suppression at the highest energies, i.e. the differentiation between the energy loss effects due to propagation, and the maximum energy of particles injected by astrophysical sources.
- Search for a flux contribution of protons up to the highest energies. We aim to reach a sensitivity to a contribution as small as $10 \%$ in the flux suppression region. The measurement of the fraction of protons is the decisive ingredient for estimating the physics potential of existing and future cosmic-ray, neutrino, and gammaray detectors; thus prospects for proton astronomy with future detectors will be clarified. Moreover, the flux of secondary gamma-rays and neutrinos due to proton energy loss processes will be predicted.

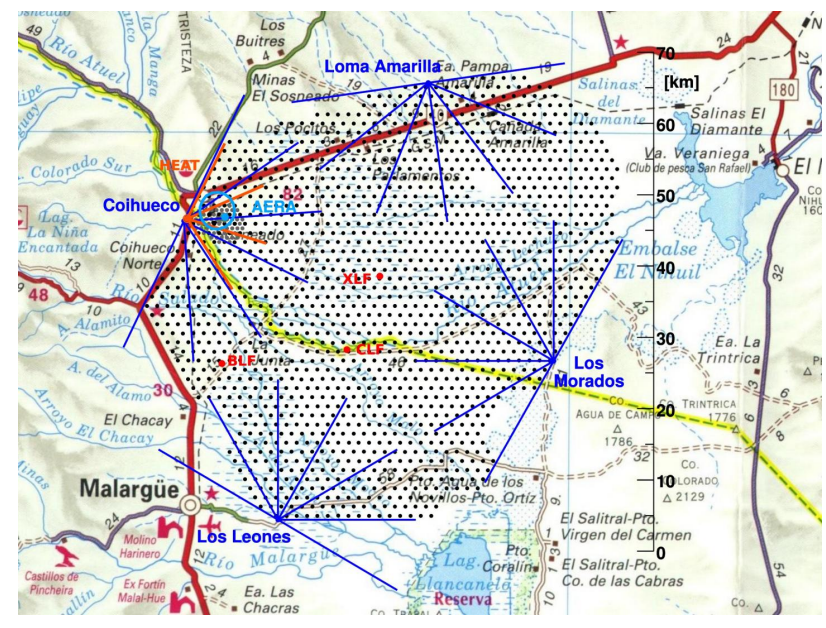

Figure 1. The Auger Observatory [1]. Each dot corresponds to one of the 1661 Surface Detector stations. The four fluorescence detector sites are illustrated, each with the $30^{\circ}$ field of view of its six telescopes. Also shown are the two laser facilities, CLF and XLF, near the center of the Observatory. In front of the Coihueco fluorescence detector is the location of the dense array, where Surface Detectors are installed on a $750 \mathrm{~m}$ grid and the location of the Auger Engineering Radio Array (AERA).

\footnotetext{
*e-mail: horandel@astro.ru.nl - http://particle.astro.ru.nl

**full authorlist available at http://www.auger.org/archive/authors_2018_10.html
} 

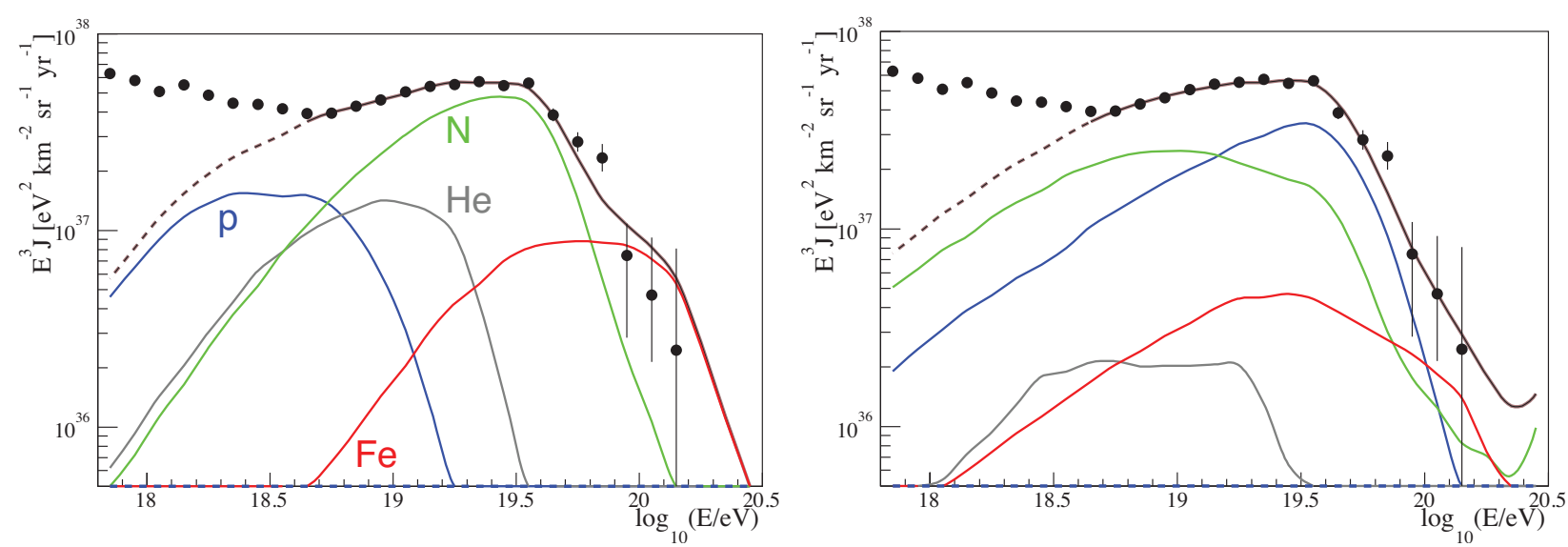

Figure 2. Examples of fluxes of different mass groups (as indicated) for describing the Auger spectrum and composition data [5]. Two scenarios are shown: particles being accelerated up to a maximum rigidity (left) and particles undergoing photo-disintegration during propagation (right).

- Study extensive air showers and hadronic multiparticle production. This will include the exploration of fundamental particle physics at energies beyond those accessible at man-made accelerators, and the derivation of constraints on new physics phenomena, such as Lorentz invariance violation or extra dimensions.

A key to understanding the origin of the highestenergy cosmic rays is to precisely measure the elemental composition up to the highest energies $\left(10^{20} \mathrm{eV}\right.$ and above). Two baseline scenarios are illustrated in Fig. 2, showing the expected energy spectra for elemental groups. The left-hand side illustrates expectations for a scenario in which cosmic rays are accelerated up to a maximum rigidity. The elemental groups exhibit fall-offs proportional to their elemental charge $Z$. The right-hand side shows an expectation for particles undergoing photo-disintegration during propagation. In this scenario a significant fraction of light particles (protons) are expected even at the highest energies, this is the main difference to the maximum rigidity scenario, in which heavier particles (large $Z$ ) are expected at the highest energies.

To achieve these objectives, a layer of scintillators is being installed above the water-Cherenkov detectors (the Surface Scintillator Detector), the observation time of the fluorescence detectors is being increased, and underground muon detectors are being installed in a part of the Surface Detector array. In addition, radio antennas will be added to each Surface Detector station as described below.

\section{Radio detection of (horizontal) air showers}

The radio emission from extensive air showers [6-8] is investigated at the Auger Observatory with AERA [9]. Radio emission is detected in the frequency range from 30 to $80 \mathrm{MHz}$ [10]. The antenna response is calibrated with a reference source in-situ in the field [11]. Timing synchronization is performed with a reference transmitter and us- ing radio pulses emitted from commercial airplanes [12]. A resolution of better than 2 ns has been achieved.

Measurements of the polarization of the radio signals allow us to have a closer look at the emission processes of the radiation in extensive air showers [13]. The majority of the emission in the atmosphere is found to be due to the interaction with the geomagnetic field (transverse separation of charges, geomagnetic effect) [14]. Of the order of $15 \%$ of the radiation is due to the longitudinal separation of charges (Askaryan effect) [15].

The renaissance of radio detection of cosmic rays began in the early 2000s [6-8]. In parallel with the experimental activities, strong efforts were devoted to the modeling of the radio emission physics. Two lines of research contributed most to the detailed understanding we have today. On the one hand, macroscopic, semianalytical calculations [16-21] revisited and refined the original concepts for geomagnetic [14] and charge-excess emission [15] in an atmosphere with a realistic refractive index gradient, thereby providing important insights into the relevant mechanisms. On the other hand, microscopic Monte Carlo simulations in the atmosphere [22-27], building on experience in calculations for showers in dense media [28], allowed detailed and parameter-free calculations of the radio signals. The measurement of the radio footprint of an air shower yields a precise determination of the shower energy [29, 30]. A cosmic ray with an energy of $1 \mathrm{EeV}$ delivers about $15.8 \mathrm{MeV}$ of energy to the ground in the frequency range from 30 to $80 \mathrm{MHz}$. The radio emission measured on the ground $E_{30-80 \mathrm{MHz}}$ can be used to establish an absolute calibration of the energy scale $E_{\mathrm{CR}}$, using the universal formula

$$
\begin{aligned}
E_{30-80 \mathrm{MHz}}=[15.8 & \pm 0.7(\text { stat }) \pm 6.7(\text { syst })] \mathrm{MeV} \\
& \times\left(\sin \alpha \frac{E_{\mathrm{CR}}}{10^{18} \mathrm{eV}} \frac{B_{\text {Earth }}}{0.24 \mathrm{G}}\right)^{2},
\end{aligned}
$$

knowing the magnetic field $B_{\text {Earth }}$ at the location of the detector and the angle $\alpha$ between the direction of the Earth magnetic field and the direction of the air shower. 


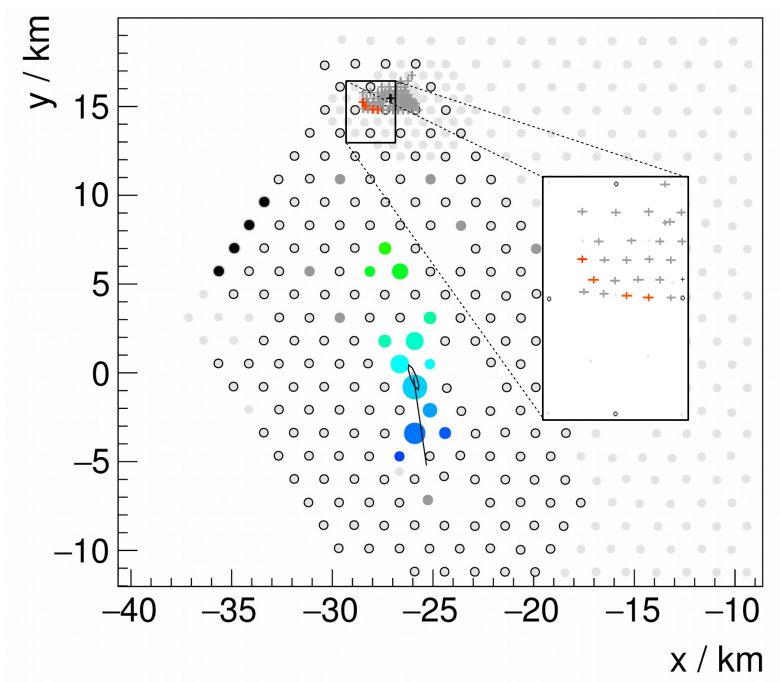

Figure 3. A measured horizontal air shower. The blue to green circles indicate the measurement with the Surface Dector, their sizes indicating energy deposit and the color encoding arrival times. Dark-grey circles indicate isolated particle detections rejected in the reconstruction. The radio signal extends over a significantly larger area than the particle distribution [31].

AERA has also been used to measure radio emission from air showers with large zenith angles between $60^{\circ}$ and $84^{\circ}$ ("horizontal air showers") [31, 32]. An interesting air shower is shown in Fig. 3. The air shower has been detected with four antennas at the edge of AERA. Its readout was triggered because an isolated Surface Detector station with significant energy deposit (dark-grey circles in Fig. 3) was closer than the $5 \mathrm{~km}$ maximum readout distance, presently set in the AERA data acquisition. The locations of the antennas with a signal (marked in red) are in alignment with the ground projection of the air-shower axis reconstructed from the Surface Detector data. The azimuth angles reconstructed from the radio signals and particle-detector measurements agree to within better than $0.5^{\circ}$. The zenith angle reconstructed with the particle detectors amounts to $83^{\circ}$, while the zenith angle determined from the arrival times of the radio signals corresponds to $87^{\circ}$. The low number of radio antennas with signal and their approximate alignment along a line perpendicular to the air-shower axis limit the zenith-angle resolution of the radio measurement in this particular case. The maximum axial distance at which a signal has been measured amounts to $2150 \mathrm{~m}$ and the exceptionally large ground distance of the measured radio signals more than $15 \mathrm{~km}$ away from the shower axis arises from projection effects. Nevertheless, this example illustrates that the ground area illuminated by radio signals can be significantly larger than the "particle footprint" on the ground.

Large footprints for horizontal air showers are also expected from simulations [7]. Simulated footprints of the radio emission of extensive air showers with various zenith angles in the $30-80 \mathrm{MHz}$ frequency band are shown in Fig. 4 for an air shower with an energy of $5 \cdot 10^{18} \mathrm{eV}$. The detection threshold governed by Galactic noise typically

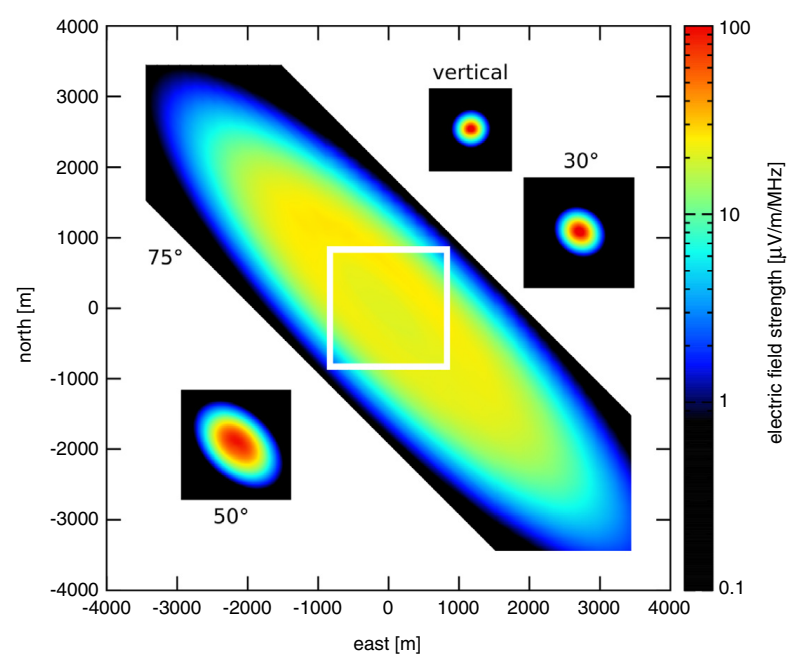

Figure 4. Simulated footprints of the radio emission of extensive air showers with various zenith angles in the $30-80 \mathrm{MHz}$ frequency band for air showers with an energy of $5 \cdot 10^{18} \mathrm{eV}$. The insets show results for different zenith angles, drawn to scale with the main figure. The detection threshold governed by Galactic noise typically corresponds to $1-2 \mathrm{mV} / \mathrm{m} / \mathrm{MHz}$ [7].

corresponds to $1-2 \mathrm{mV} / \mathrm{m} / \mathrm{MHz}$. The footprint is small for air showers with zenith angles up to $60^{\circ}$, but becomes very large for inclined showers with zenith angles of $70^{\circ}$ or higher. The white rectangle denotes the size of the $50^{\circ}$ inset. The strong increase of the area illuminated by inclined air showers is due to the large geometrical distance of their emission region from the ground, as sketched in the inset in Fig. 5.

The size of the radio footprint of an air shower has been measured. The distance from the shower axis to the radio detector furthest away (in the shower plane, perpendicular to the shower axis) is depicted in Fig. 5 as a function of the zenith angle of the air shower. In the figure also the expected number of radio stations (on the standard Auger $1500 \mathrm{~m}$ grid) with a signal above threshold are indicated together with an estimate of the size of the footprint on the ground. As can be seen, for horizontal air showers (i.e. large zenith angles) the footprints reach sizes exceeding tens of $\mathrm{km}^{2}$ and dozens of stations will have a signal above the threshold. This is a very important result since it confirms experimentally that the radio emission from horizontal air showers can be measured with radio antennas on the standard Auger $1500 \mathrm{~m}$ grid.

\section{The Auger radio upgrade}

We are going to install a radio antenna on each of the 1661 stations of the Surface Detector array of the Observatory (see Fig. 1), forming a $3000 \mathrm{~km}^{2}$ radio array, the largest radio array for cosmic-ray detection in the world. An artist's impression of the planned set-up is given in Fig. 6. It shows (from bottom to top) the water-Cherenkov detector with a layer of scintillators on top (Surface Scintillator Detector) and a radio antenna (Radio Detector). The 


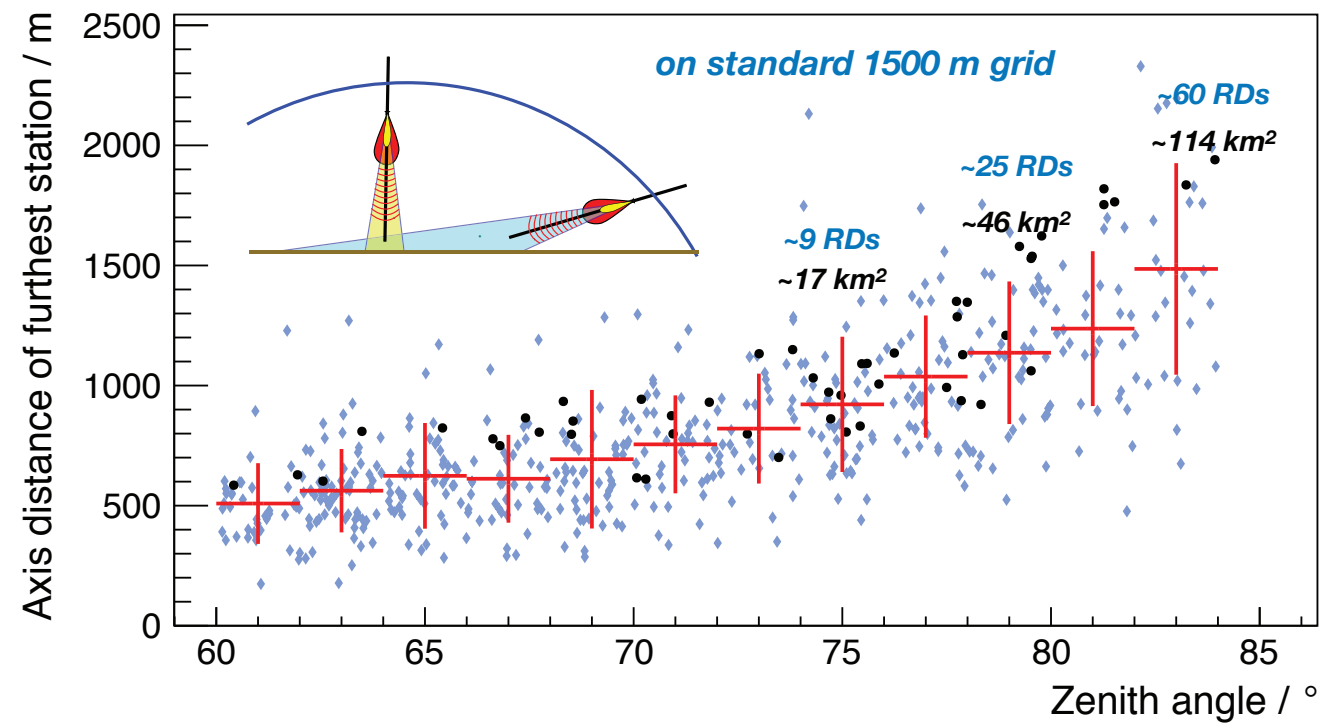

Figure 5. Furthest axis distance at which a radio signal above noise background has been detected with AERA as a function of the air-shower zenith angle [31].

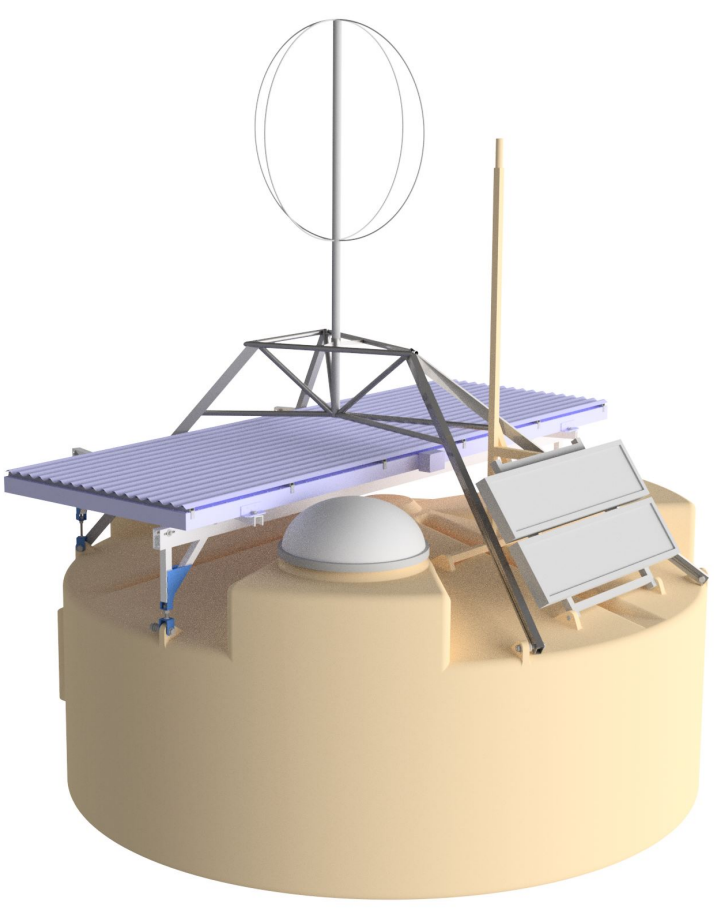

Figure 6. Conceptional illustration of an upgraded station of the Surface Detector array of the Pierre Auger Observatory, comprised of (from bottom to top) a water-Cherenkov detector, a layer of scintillators, and a radio antenna.

concept of radio antennas on top of the Surface Detector stations has been studied earlier at the Auger Observatory on smaller scales $[33,34]$. With the combination of water-Cherenkov detector and Surface Scintillator Detector the electron-to-muon ratio $(\mathrm{e} / \mu)$ is measured for vertical showers. In a similar way the combination of water-
Cherenkov detector and Radio Detector will be used to measure the ratio of the electromagnetic energy and the number of muons for horizontal air showers. In turn, the $\mathrm{e} / \mu$ ratio will be used to derive the particle type of the incoming cosmic ray up to the highest energies. This is the main goal of the Auger upgrade, to measure the particle type of each incoming cosmic ray. Thus, the radio upgrade will increase the aperture of the Observatory for mass-sensitive investigations, allowing the e/ $\mu$ separation for showers with a broad zenith angle range, from zenith with the Surface Scintillator Detector to the horizon with the Radio Detector.

There is an important difference related to the mass measurement of cosmic rays with AERA (for vertical showers) and the radio upgrade (for horizontal air showers). For vertical showers we use a geometrical method, correlating the size of the footprint on the ground to the distance from the observer to the shower maximum $[2,3,35]$. From this quantity, the depth of the shower maximum $X_{\max }$ is derived, which in turn is dependent on the logarithm of the number of nucleons (nuclear mass), $A$, of the incoming cosmic ray $X_{\max } \propto \ln A$ [2]. For horizontal showers, we aim to apply a different method: We will use a combination of radio antennas and the water-Cherenkov detectors to measure the $\mathrm{e} / \mu$ ratio in air showers to determine $\ln A$.

Two effects are important for the detection of horizontal air showers with the radio upgrade. First, the enlarged footprint of the radio emission, as explained above, and, second, the absorption behavior of air showers in the atmosphere, as sketched in Fig. 7. For horizontal air showers the electromagnetic as well as the hadronic components are absorbed in the atmosphere and only the muons reach the detector. The atmosphere is transparent to radio emission in the frequency band $30-80 \mathrm{MHz}$, thus, the radiation (most of it being produced near the shower 


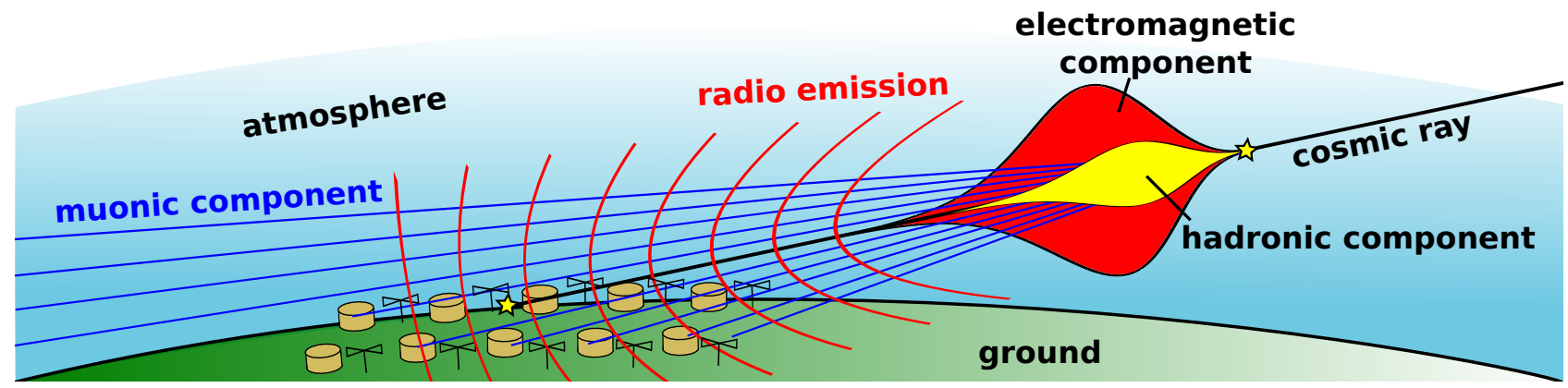

Figure 7. Sketch of a horizontal air shower. The electromagnetic as well as the hadronic air shower components are absorbed in the atmosphere and only muons penetrate to the detectors. The atmosphere is transparent to radiation with frequencies in the $30-80 \mathrm{MHz}$ band. (courtesy Ewa Holt)
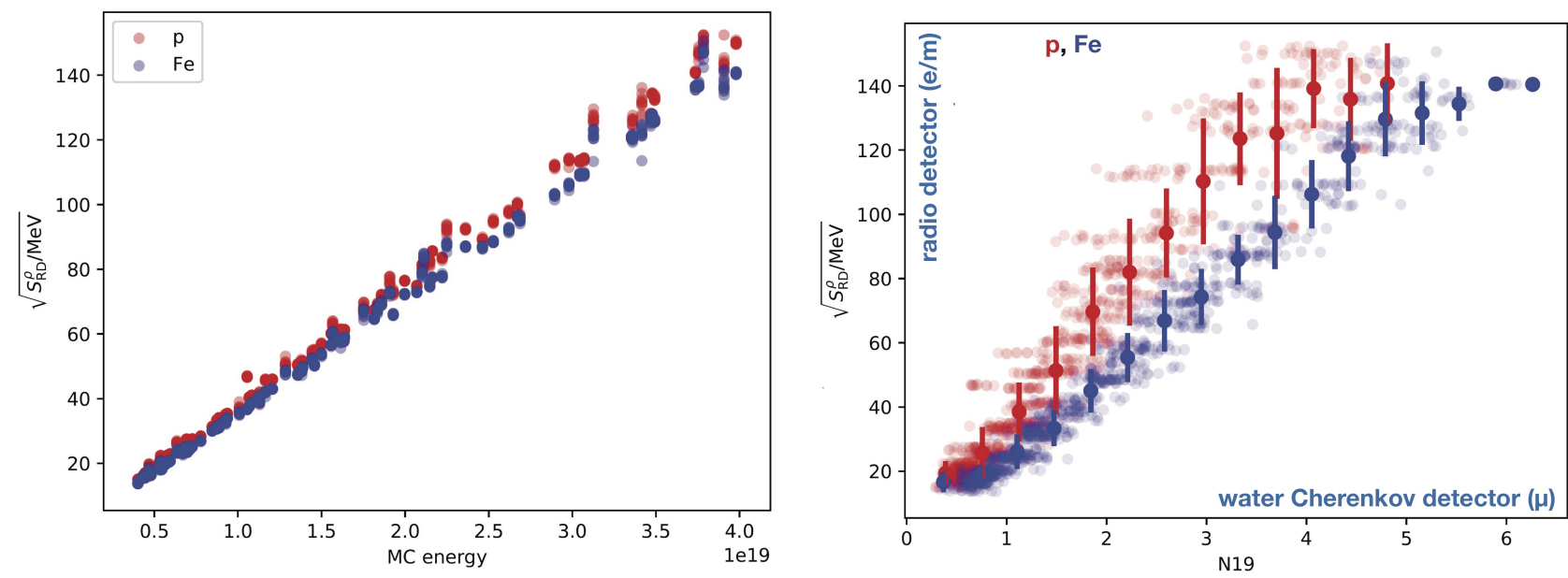

Figure 8. Estimated performance of the Radio Detector. Preliminary Results; The radio detector resolution is not yet included. Left: radiation energy (i.e. the electromagnetic energy of the shower) as a function of the shower energy. Right: electromagnetic energy in a shower as measured by the Radio Detector as a function of the muon content in a shower as measured by the water-Cherenkov detector.

maximum) penetrates the atmosphere undisturbed. This implies that one obtains a clean measurement of the electromagnetic shower energy with the Radio Detector and a clean measurement of the muonic component with the water-Cherenkov detector.

Increasing the mass sensitivity of the Observatory is crucial to understand the origin of cosmic rays, e.g. a good mass sensitivity is needed to distinguish between the baseline scenarios illustrated in Fig. 2. Precise mass (and charge $Z$ ) information will also allow to constructing more meaningful sky maps using intervals of rigidity $E / Z$ in place of energy $E$ (as done so far). Isolating particles with a small (or no) charge such as protons, neutrinos, and gamma rays is important to conduct astronomy with such particles at energies exceeding EeV energies. This will give direct hints to the cosmic-ray sources. Finally, the Radio Detector will provide a clean measurement of the electromagnetic $(\mathrm{e} / \mathrm{m})$ component of the air showers. This is of interest to study shower physics in the atmosphere. In particular, in the densely populated part of the Surface Detector array [5], we will have water-Cherenkov detectors and Surface Scintillator Detectors with a $750 \mathrm{~m}$ spacing, underground muon detectors (AMIGA), and also radio antennas. We will have a clean measurement of the e/m component from the Radio Detector and a clean measurement of the muons with AMIGA. This will allow us to cross check the unfolding of the e/ $\mu$ ratio with the Surface Scintillator Detector-water-Cherenkov detector combination, based on measured showers. Last but not least, the Radio Detector will provide an independent mass and energy scale to cross check the Surface Scintillator Detectorwater-Cherenkov detector results [29, 30].

\subsection{Performance}

In a first analysis the physics potential of the radio upgrade has been studied using CORSIKA[36]/CoREAS[22] simulations. A total of 192 showers has been simulated, half of them protons, half iron nuclei, with energies from 4 to $40 \mathrm{EeV}$ and zenith angles between $60^{\circ}$ and $80^{\circ}$. A full water-Cherenkov detector simulation and reconstruction has been included in the analysis, but only a simplified treatment of the radio signals was available. The corrected radiation energy has been calculated as described in [37]. The radiation energy has been "smeared out" in order to mimic reconstruction uncertainties. Preliminary 


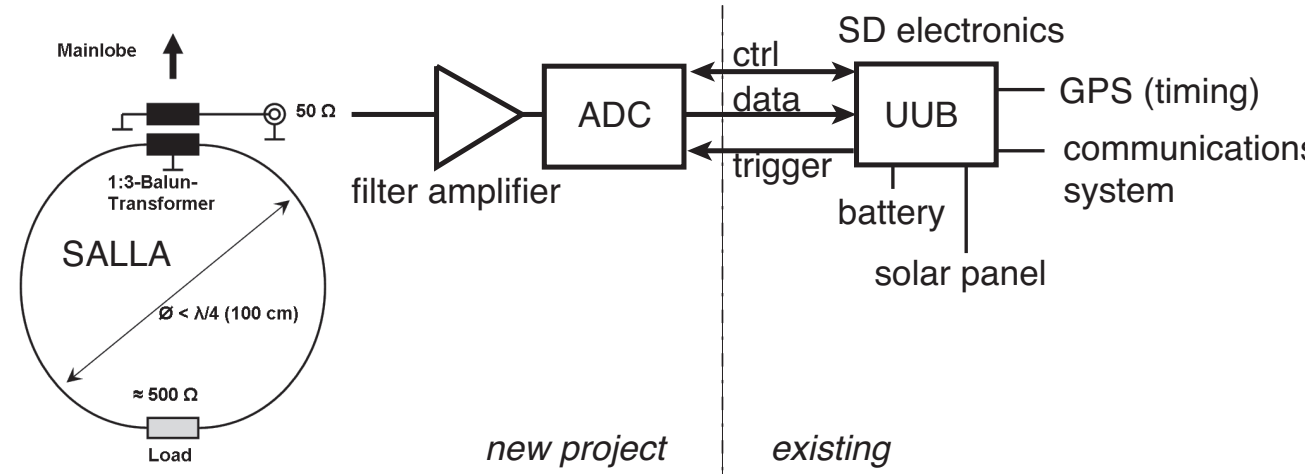

Figure 9. Schematic view of the envisaged read out for the radio antennas. The radio antenna (here illustrated as "SALLA") is read out through a filter amplifier and an ADC. The front-end board has an interface to the existing electronics (UUB) at each Surface Detector station.

results are illustrated in Fig. 8. The left-hand panel depicts the detected radiation energy as a function of the shower energy. A clear correlation can be seen and only a small dependence on the mass of the incoming cosmic rays (represented in the figure through the extrema protons and iron nuclei). The mass-sensitivity is illustrated on the righthand side. The size of the $\mathrm{e} / \mathrm{m}$ component (as measured with the Radio Detector) is plotted as a function of the muon number (as obtained with the water-Cherenkov detector). A clear separation between showers induced by protons and iron nuclei is visible. Please note: the flattening at the top-right is due to an artifact, only showers up to $40 \mathrm{EeV}$ have been simulated. This is work in progress. We are working on more precise simulation studies in order to quantify various properties of the radio upgrade such as mass resolution, energy resolution, effective aperture as a function of energy, etc.

\subsection{Technical implementation}

At present we are working on the details of the technical implementation of the radio upgrade. The Radio Detector will be fully integrated in the Surface Detector stations, they will form one unit, being comprised of waterCherenkov detector, Surface Scintillator Detector, and Radio Detector. The different detectors will share the infrastructure such as solar power, battery, communications system, GPS timing, and an integrated data acquisition system. The envisaged system is schematically shown in Fig. 9.

We aim to use a short aperiodic loaded loop antenna (SALLA) to detect the radio emission from air showers in the frequency range 30 to $80 \mathrm{MHz}$. The SALLA realizes a Beverage antenna as a dipole loop of $1.2 \mathrm{~m}$ diameter [10]. The SALLA has been developed to provide a minimal design that fulfills the need for both, ultra-wideband sensitivity, and low costs for production and maintenance of the antenna in a large-scale radio detector. The compact structure of the SALLA makes the antenna robust and easy to manufacture. A picture of a SALLA, showing its two polarization planes, is displayed in Fig. 12 below.

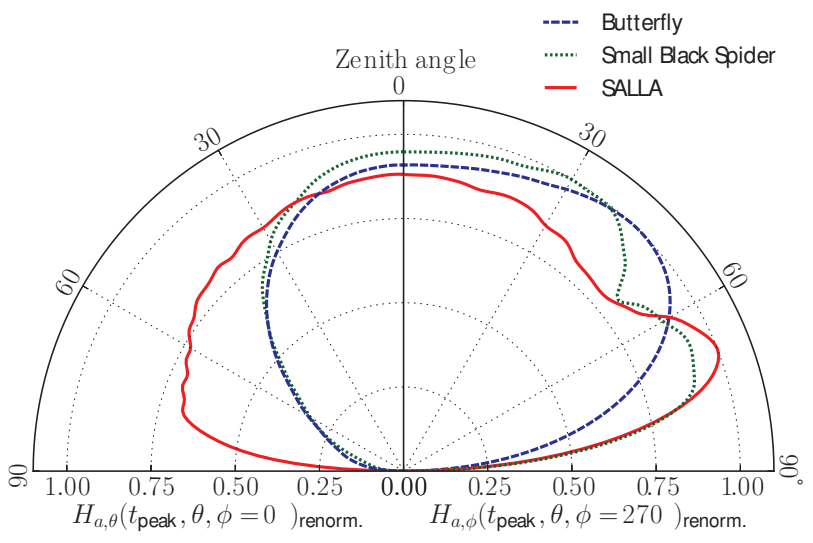

Figure 10. Peak directional diagram for the SALLA antenna together with the two antenna types installed at AERA, the logarithmic periodic dipole antenna (Small Black Spider) and the butterfly antenna: the development of the maximum absolute value of the vector effective length (VEL) in the time domain is displayed as a function of zenith angle. The two components of the VEL are treated separately. The zenith angle dependence of the $\vec{e}_{\theta}$-component of the VEL is shown in the left half of the diagram. In the right half the $\vec{e}_{\phi}$-component is depicted. Two azimuthal directions have been chosen to maximize the readings of the respective VEL component. For details, see [10].

Beverage antennas include a resistor load within the antenna structure to give a specific shape to the directivity [10]. In the case of the SALLA a resistance of $500 \Omega$ connects the ends of the dipole arms at the bottom of the antenna. The antenna is read out at the top which is also the position of the Low-Noise Amplifier (LNA). While signals coming from above will induce a current directly at the input of the amplifier, the reception from directions below the antenna is strongly suppressed as the captured power is primarily consumed within the ohmic resistor rather than amplified by the LNA. The resulting strong suppression of sensitivity towards the ground reduces the dependence of the antenna on environmental conditions which might vary as a function of time and are thus a source of systematic uncertainty. With the inclusion of an ohmic resistor the SALLA especially challenges its amplifier as only $\sim 10 \%$ 


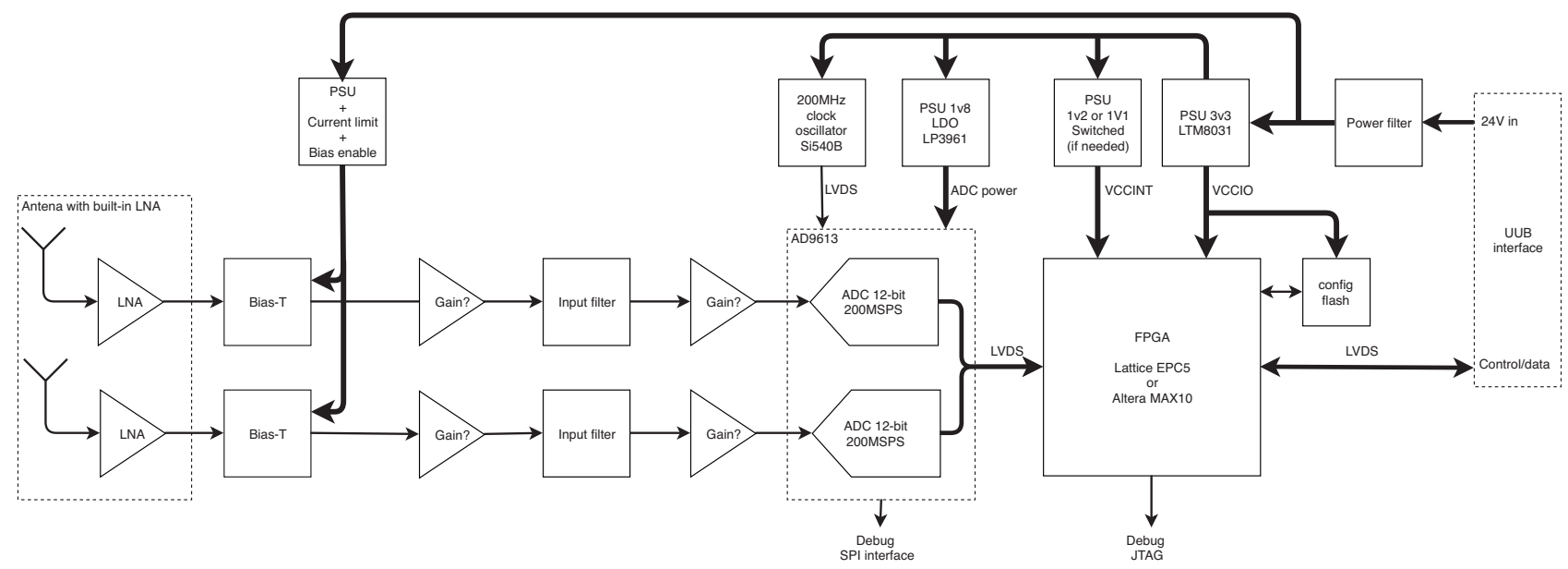

Figure 11. Block diagram of the front-end board for the radio detectors (draft - work in progress).

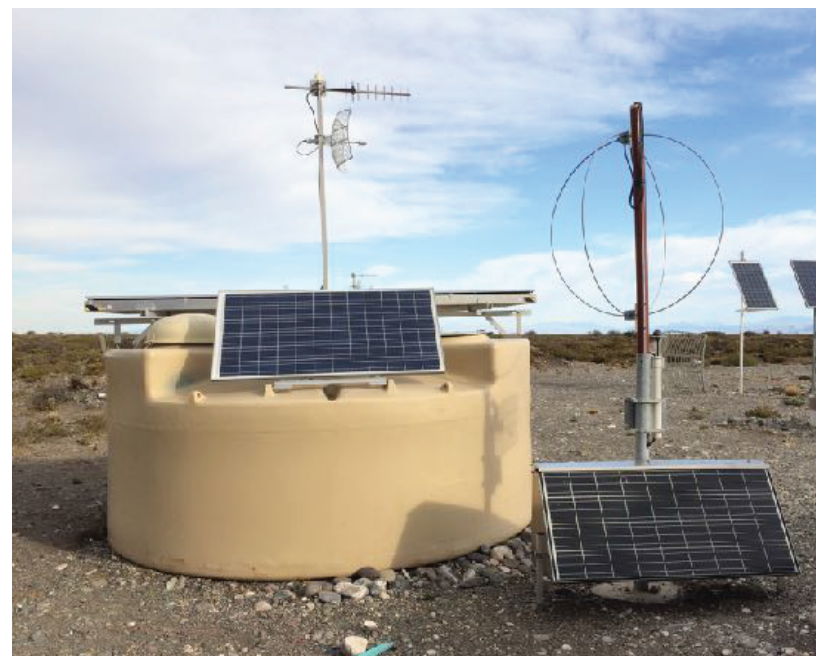

Figure 12. A prototype Radio Detector station (front, right). The SALLA antenna is visible with its two antenna rings with a diameter of $1.2 \mathrm{~m}$. On the left a Surface Detector station of the Pierre Auger Observatory with a Surface Scintillator Detector module on top of a water-Cherenkov detector.

of the captured signal intensity is available at the input of the LNA. Proper matching between the antenna structure and the LNA is realized with a 3:1 transmission line transformer. The structure of the SALLA creates a sensitivity which is flat as a function of frequency.

The antenna response has been characterized in field measurements [10] and the vector effective length has been determined as a function of the zenith angle over our frequency band from 30 to $80 \mathrm{MHz}$. The results are shown in Fig. 10 for the SALLA antenna together with the antennas installed at AERA. The SALLA antenna exhibits a good sensitivity for large zenith angles. This is an important aspect for the application in the radio upgrade. Also longterm experience is available, about 60 SALLA antennas are already used at the Tunka site to measure air showers on a regular basis [38].

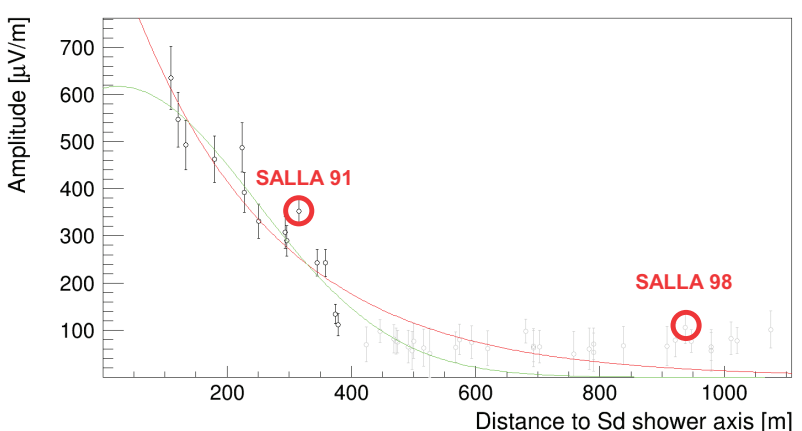

Figure 13. Lateral distribution function of the radio emission from an air shower as measured with AERA and two prototype antennas (indicated as "SALLA").

The water-Cherenkov detector will issue a trigger signal when energy deposition has been detected. The data from the radio antenna will be passed to the readout electronics of the Surface Scintillator Detector/waterCherenkov detector system (UUB) and will be transmitted together with all data from the station to the central data acquisition of the Auger Observatory.

A schematic view of the radio front-end board is depicted in Fig. 11. We foresee two polarization directions of the antenna, oriented orthogonal to each other (see also Fig. 12). The signals of the two analogue channels will be pre-amplified in a LNA at the antenna. The signals are transmitted through shielded coaxial cables to the filter amplifier on the front-end board. They will be digitized with a sampling frequency of 200 Msps. A FPGA controls the data flow and the communication with the existing electronics of each Surface Detector station (UUB).

To test the performance of the SALLA antennas we have installed three prototype stations inside the AERA field at the Auger Observatory. Such a prototype station is shown in Fig. 12; An AERA station with SALLA antenna is situated next to a water-Cherenkov detector with a Surface Scintillator Detector module on top. 


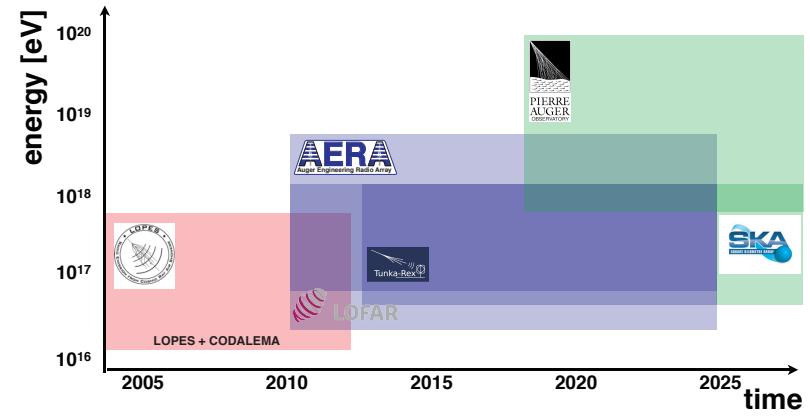

Figure 14. Schematic view of the development of the radio detection technique. The approximate energy range for various experimental set-ups is plotted versus the approximate operation time of those instruments.

First air showers have been measured in coincidence with the prototype stations. As an example, the amplitude of the radio signal for a measured air shower is presented in Fig. 13 as a function of the distance to the shower axis. The signals from the SALLA antennas are compared to the "regular" AERA stations. The signals measured with the SALLA antennas are slightly bigger, due to a preliminary calibration used for the SALLA antennas.

\subsection{Schedule}

Our plan for the installation of the radio upgrade at the Auger Observatory is as follows. We are presently working on the details of the technical implementation. We aim to conclude this throughout the year 2019. We envisage starting the mass production of the detector components in the second half of 2019 and are looking forward to deploying the detectors in the field in 2020.

The development of the radio detection technique is illustrated schematically in Fig. 14. For various experimental set-ups the approximate energy range and the approximate time of operation are indicated. The modern era of the radio detection of air showers started around the year 2000. Within two decades the experiments grew in size from about $0.1 \mathrm{~km}^{2}$ to now $3000 \mathrm{~km}^{2}$. As a consequence, the energy range of interest shifted from a few $10^{16} \mathrm{eV}$ to above $10^{20} \mathrm{eV}$. With the upgraded Auger Observatory and its $3000 \mathrm{~km}^{2}$ radio array we will be able to investigate the properties of cosmic rays up to the highest energies, exceeding $10^{20} \mathrm{eV}$. This will be a milestone in the development of the radio technique to measure extensive air showers and the investigation of the origin of the highest-energy cosmic rays.

\section{Acknowledgement}

We appreciate funding from the BMBF/Germany to conduct $R \& D$ for this project. The realization of the radio upgrade is made possible through funding from the European Research Council (ERC) under the European Union's Horizon 2020 research and innovation programme (grant agreement No 787622, ERC Advanced Grant Hörandel) and the Netherlands Organisation of Scientific Research (NWO).

\section{References}

[1] A. Aab et al. (Pierre Auger Collaboration), Nucl. Instrum. Meth. A798, 172 (2015).

[2] J. Schulz et al. (Pierre Auger Collaboration), Proceedings 34th International Cosmic Ray Conference, Den Haag PoS(ICRC2015)615 (2015)

[3] T. Huege et al. (Pierre Auger Collaboration), Proceedings UHECR symposium, Paris (2018)

[4] A. Castellina et al. (Pierre Auger Collaboration), Proceedings UHECR symposium, Paris (2018)

[5] A. Aab et al. (Pierre Auger Collaboration), arXiv:1604.03637 (2016).

[6] J. R. Hörandel, Proceedings ARENA 2018 (2018)

[7] T. Huege, Phys. Rept. 620, 1 (2016).

[8] F. G. Schröder, Prog. Part. Nucl. Phys. 93, 1 (2017).

[9] P. Abreu et al. (Pierre Auger Collaboration), Nucl.Instrum.Meth. A635, 92 (2011).

[10] P. Abreu et al. (Pierre Auger Collaboration), JINST 7, P10011 (2012).

[11] A. Aab et al. (Pierre Auger Collaboration), JINST 12, T10005 (2017).

[12] A. Aab et al. (Pierre Auger Collaboration), JINST 11, P01018 (2016).

[13] A. Aab et al. (Pierre Auger Collaboration), Phys.Rev. D89, 052002 (2014).

[14] F. Kahn, I. Lerche, Royal Society of London Proceedings Series A 289, 206 (1966)

[15] G. Askaryan, Journal of the Physical Society of Japan Supplement 17, C257 (1962)

[16] K. Werner, O. Scholten, Astropart. Phys. 29, 393 (2008).

[17] O. Scholten, K. Werner, F. Rusydi, Astropart. Phys. 29, 94 (2008).

[18] O. Scholten, K. D. de Vries, K. Werner Nucl. Instrum. Meth. A662, S80 (2012).

[19] K.D. de Vries, A. M. van den Berg, O. Scholten, K. Werner, Phys. Rev. Lett. 107, 061101 (2011).

[20] K. Werner, K. D. de Vries, O. Scholten, Astropart. Phys. 37, 5 (2012).

[21] O. Scholten, T.N.G. Trinh, K.D. de Vries, B. M. Hare, Phys. Rev. D97, 023005 (2018).

[22] T. Huege, M. Ludwig, C. W. James, ARENA 2012, AIP Conf. Proc. 1535 pp. 128-132 (2013)

[23] M. Ludwig, T. Huege, Astropart. Phys. 34, 438 (2011).

[24] C. W. James, H. Falcke, T. Huege, M. Ludwig, Phys. Rev. A84, 056602 (2011).

[25] J. Alvarez-Muñiz, W. R. Carvalho, Jr., E. Zas, Astropart. Phys. 35, 325 (2012).

[26] V. Marin, B. Revenu, Astropart. Phys. 35, 733 (2012).

[27] J. Alvarez-Muñiz, W. R. Carvalho, A. Romero-Wolf, M. Tueros, E. Zas, Phys. Rev. D86, 123007 (2012). 
[28] E. Zas, F. Halzen, T. Stanev, Phys. Rev. D45, 362 (1992)

[29] A. Aab et al. (Pierre Auger Collaboration), Phys. Rev. D93, 122005 (2016).

[30] A. Aab et al. (Pierre Auger Collaboration), Phys. Rev. Lett. 116, 241101 (2016).

[31] A. Aab et al. (Pierre Auger Collaboration), JCAP 1810, 026 (2018).

[32] M. Gottowik et al., Proceedings ARENA 2018 (2018)

[33] C. Berat for the Pierre Auger Collaboration, Nucl. Instr. Meth. A 718, 471 (2013).
[34] R. Gaïor, Easier: extensive air shower identification using electron radiometer, https://hal.archivesouvertes.fr/tel-01826441.

[35] S. Buitink, et al., Phys.Rev. D90, 082003 (2014).

[36] D. Heck et al., CORSIKA: A Monte Carlo code to simulate extensive air showers, Report FZKA 6019, Forschungszentrum Karlsruhe (1998)

[37] C. Glaser, M. Erdmann, J. R. Hörandel, T. Huege, J. Schulz, JCAP 1609, 024 (2016).

[38] F. G. Schröder et al., JPS Conf. Proc. 9, 010008 (2016). 